\title{
Uniqueness Theorems for Willmore Surfaces with Fixed and Free Boundaries
}

\author{
BENNETT PALMER
}

\begin{abstract}
We prove that a Willmore surface of disc type which has its boundary on a circle and which intersects the plane of the circle in a constant angle is a spherical cap or a flat disc. We prove the existence of disc type Willmore surfaces with circular boundary which intersect the plane of the circle in a nonconstant angle. We formulate and prove a uniqueness theorem for Willmore surfaces in a half space with free boundary in a plane.
\end{abstract}

\section{INTRODUCTION}

Recently there has been some activity in trying to determine the compact constant mean curvature surfaces in $\mathbb{E}^{3}$ having their boundary on a round circle. Kapouleas [7] showed the existence of examples of genus greater than one so such a surface is not necessarily a part of a round sphere. Even with the additional hypothesis that the surface is embedded or that the surface has genus zero, it is not known at the present time if the surface need be a spherical cap or disc. Partial results can be found in [19], [10], [5], [1].

The problem mentioned above might be considered as part of the more general question: Does a solution of a variational problem necessarily inherit the symmetries of its boundary? We are assuming, of course, that the symmetries of the boundary are also symmetries of the variational problem. Here we will treat a version of this problem for Willmore surfaces. Here we will say that a Willmore surface is a sufficiently smooth immersed surface $X: \Sigma \rightarrow \mathbb{E}^{3}$ which is a critical point of the functional

$$
W[X]=\int_{\Sigma} h^{2}-K d A
$$

with respect to compactly supported variations of the surface. Throughout this paper $h$ and $K$ will denote the mean and Gauss curvatures. 
Although boundary value problems for Willmore surfaces have not been widely studied, (notable exceptions are [13], [14]), they are of some physical interest. For example, the (unlinearized) potential energy density of a plate given in CourantHilbert ([4], page 250) is variationally equivalent, when the plate is clamped, to the Willmore energy.

The Euler-Lagrange equation for $W$ is

$$
\Delta h+2 h\left(h^{2}-K\right)=0 .
$$

Note that since $h$ depends on second derivatives of $X$, this is a fourth order equation. Equation (1.1) was first found in its nonparametric form by Poisson [16] in connection with elasticity theory and its invariant form is due to Schadow.

In order to state our result let $S^{1}$ denote the unit circle in the plane $\left\{x_{3}=0\right\}$.

Theorem 1.1. Let $D$ denote the unit disc and let $X:(D, \partial D) \rightarrow\left(\mathbb{E}^{3}, S^{1}\right)$ be a $C^{4}(\bar{D})$ immersion of a Willmore surface. Assume that the surface intersects the plane $\left\{x_{3}=0\right\}$ in a constant angle along $\partial D$. Then the image $X(D)$ is a spherical cap or a flat disc.

The inclusion of the hypothesis of constant angle between the surface and the plane along the boundary is justified by the order of Equation (1.1). Using the inverse function theorem, we will show below that there exist infinitely many Willmore surfaces of disc type with circular boundary and which intersect the plane of the circle in a nonconstant angle. On the other hand, our hypotheses are quite natural since they are invariant under conformal transformations of $\mathbb{E}^{3}$ as is the functional $W$. It should be noted that the main tool for obtaining uniqueness results for CMC surfaces is the maximum principle which has no known counterpart for Willmore surfaces.

We wish to point out a relevant example of Babich and Bobenko [2] of a Willmore surface which is a surface of revolution having the topology of the disc. However this example has a singularity at the origin. Pictures of the surface may be found in [2], figures $2 \mathrm{~A}$ and $2 \mathrm{~B}$.

If we consider the variational problem of extremizing the functional $W$ with respect to all variations which pointwise fix the boundary, then we arrive at the natural boundary condition that the mean curvature equals the normal curvature along the boundary. In Section 5 we obtain a uniqueness theorem for embedded disc type Willmore surfaces with circular boundary under this boundary condition. We wish to thank the referee for suggesting this problem.

The remaining parts of the paper is concerned with a free boundary problem for Willmore surfaces. We formulate the necessary and sufficient conditions that a smooth, compact surface be a critical point for the Willmore functional among all surfaces contained in a domain $\Omega \subset \mathbb{E}^{3}$ whose boundaries are constrained to lie on $\partial \Omega$. These conditions were previously obtained in another form (and in greater generality) by Nitsche [13]. We include our derivation for clarity and completeness. Inspired by another paper of Nitsche [12] on the free boundary 
problem for the area functional, we show that in the case $(\Omega, \partial \Omega)=\left(\mathbb{E}_{+}^{3}, \mathbb{E}^{2}\right)$, the only critical immersions of disc type for the aforementioned variational problem are totally umbilic. The same result holds for $(\Omega, \partial \Omega)=\left(B, S^{2}\right)$ where $B$ is the unit ball.

We conclude with a remark on terminology. Usually the Willmore functional is defined by

$$
\bar{W}[X]=\int_{\Sigma} h^{2} d A .
$$

For surfaces without boundary the functionals $W$ and $\bar{W}$ are variationally equivalent. However, for surfaces with boundary the inclusion of the curvature term may make a difference depending on the chosen boundary conditions. For clamped boundary, i.e. fixing the boundary to first order, $W$ and $\bar{W}$ are variationally equivalent. However, for the type of problems discussed in Sections 5 and 6, they are not. We have chosen to prefer to use $W$ because of the conformal invariance of the integrand.

\section{PRELIMINARIES}

Let $X:(D, \partial D) \rightarrow\left(\mathbb{E}^{3}, S^{1}\right)$ be any $C^{1}(\bar{D})$ immersion. We orient $\partial D$ in the usual way (counterclockwise) and let $t$ and $n$ respectively denote the the tangent and outward pointing conormal defined along the boundary. We let $v$ the unit normal field on $D$ such that $n \times t=v$ holds on $\partial D$.

Let $E_{3}$ denote the vertical unit vector in $\mathbb{E}^{3}$. If we define an angle $\gamma$ by $\cos \gamma:=$ $\langle X, v\rangle$ then it is easy to check that

$$
\begin{gathered}
\langle X, v\rangle=\cos \gamma=-\left\langle n, E_{3}\right\rangle, \\
\langle X, n\rangle=\sin \gamma=\left\langle v, E_{3}\right\rangle
\end{gathered}
$$

hold.

An important tool for working with Willmore surfaces is the conformal Gauss map. Let $S_{1}^{4}$ denote the deSitter space $S_{1}^{4}:=\left\{Y \in \mathbb{E}_{1}^{5} \mid Y \cdot Y=1\right\}$. The space $S_{1}^{4}$ can be identified with the set of oriented 2 -spheres and oriented planes in $\mathbb{E}^{3}$. If $X: \Sigma \rightarrow \mathbb{E}^{3}$ is a smooth immersion of an oriented surface, then the conformal Gauss map $Y: \Sigma \rightarrow S_{1}^{4}$ assigns to each $p \in \Sigma$ the oriented 2-sphere in oriented contact with the surface at $X(p)$ and having mean curvature equal to the mean curvature of $\Sigma$ at $p$. If $v$ denotes the unit normal field on $\Sigma$ then $Y$ can be expressed in terms of coordinates on $\mathbb{E}_{1}^{5}$ as

$$
Y=h\left(X, \frac{X^{2}-1}{2}, \frac{X^{2}+1}{2}\right)+(v,\langle X, v\rangle,\langle X, v\rangle)
$$

Away from umbilics in $\Sigma, Y$ defines a conformal spacelike immersion from $\left(\Sigma, d s_{X}^{2}\right)$ into $S_{1}^{4}$. It was shown by G. Thomsen [18], that $\Sigma$ is a Willmore surface if and 
only if $Y$ defines a zero mean curvature immersion on $\Sigma$ minus the umbilic set. In this case we have that $Y$ satisfies

$$
\Delta Y+2\left(h^{2}-K\right) Y=0
$$

on $\Sigma$. As a consequence we have the following lemma.

Lemma 2.1. (Flux formula) Let $X: \Sigma \rightarrow \mathbb{E}^{3}$ be a Willmore immersion and let $Y=\left(Y_{1}, \ldots, Y_{5}\right)$ be its conformal Gauss map. Then for $1 \leq \alpha<\beta \leq 5$, the forms defined by

$$
\omega_{\alpha \beta}:=Y_{\alpha} * d Y_{\beta}-Y_{\beta} * d Y_{\alpha}
$$

are closed.

Proof. Compute

$$
d \omega_{\alpha \beta}=\left(Y_{\alpha} \Delta Y_{\beta}-Y_{\beta} \Delta Y_{\alpha}\right) * 1=0
$$

by $(2.5)$.

\section{Circular Boundary}

We begin by specializing the flux formula to a Willmore surface with circular boundary. We will let $k_{n}$ denote the normal curvature of the boundary curve.

Lemma 3.1. Let $\Sigma$ be a compact, oriented surface with boundary homeomorphic to a circle and let $X:(\Sigma, \partial \Sigma) \rightarrow\left(\mathbb{E}^{3}, S^{1}\right)$ be a $C^{4}(\bar{\Sigma})$ Willmore immersion. Define a (not necessarily constant) angle $\gamma$ by (2.2). Then the following hold:

$$
\begin{gathered}
\int_{\partial \Sigma} k_{n}-h d s=0 \\
\int_{\partial \Sigma} \cos (\gamma) \partial_{n} h+\sin (\gamma) h\left(h-k_{n}\right) d s=0 \\
\int_{\partial \Sigma} \sin (\gamma) \partial_{n} h-\cos (\gamma) h\left(h-k_{n}\right) d s=0
\end{gathered}
$$

Proof. We will use the following

$$
\partial_{n} v_{3}=\left\langle d v(n), E_{3}\right\rangle=\left\langle n, E_{3}\right\rangle\langle n, d v(n)\rangle=\left\langle n, E_{3}\right\rangle\left(k_{n}-2 h\right)
$$


and

$$
\partial_{n}\langle X, v\rangle=\langle X, d v(n)\rangle=\langle X, n\rangle\langle n, d v(n)\rangle=\langle X, n\rangle\left(k_{n}-2 h\right)
$$

Note that $\left.Y\right|_{\partial \Sigma}=h\left(x_{1}, x_{2}, 0,0,1\right)+\left(\nu_{1}, v_{2}, \sin \gamma, \cos \gamma, \cos \gamma\right)$. Using this, we compute on $\partial D$

$$
\begin{aligned}
\omega_{34} & =\sin \gamma * d\left(h\left(\frac{X^{2}-1}{2}\right)\right)-\cos \gamma * d\left(h x_{3}+v_{3}\right) \\
& =\left[\sin \gamma\left(h\langle X, n\rangle+\langle X, d v(n)\rangle-\cos \gamma\left(h\left\langle E_{3}, n\right\rangle+\left\langle E_{3}, d v(n)\right\rangle\right)\right] d s\right. \\
& =\left[\sin \gamma\left(h \sin \gamma-\sin \gamma\left(2 h-k_{n}\right)\right)+\cos \gamma\left(h(\cos \gamma)+\cos \gamma\left(k_{n}-2 h\right)\right)\right] d s \\
& =\left[k_{n}-h\right] d s .
\end{aligned}
$$

Next note that $y_{5}=y_{4}+h$ holds and so

$$
\begin{aligned}
\omega_{45} & =y_{4} * d h-h * d y_{4} \\
& =\cos \gamma * d h-h * d\left(h \frac{X^{2}-1}{2}+\langle X, v\rangle\right) \\
& =\left[\cos \gamma \partial_{n} h-h\left(h\langle X, n\rangle+\partial_{n}\langle X, v\rangle\right)\right] d s \\
& =\left[\cos \gamma \partial_{n} h-h\left(h\langle X, n\rangle+\langle X, n\rangle\left(k_{n}-2 h\right)\right)\right] d s \\
& =\left[\cos \gamma \partial_{n} h-h \sin \gamma\left(h+\left(k_{n}-2 h\right)\right)\right] d s \\
& =\left[\cos \gamma \partial_{n} h+h \sin \gamma\left(h-k_{n}\right)\right] d s
\end{aligned}
$$

Again using $y_{5}=y_{4}+h$ we have

$$
\begin{aligned}
\omega_{35} & =\sin \gamma * d h-h * d y_{3}+\omega_{34} \\
& =\sin \gamma * d h-h *\left(h x_{3}+v_{3}\right)+\omega_{34} \\
& =\left[\sin \gamma \partial_{n} h-h\left(h\left\langle n, E_{3}\right\rangle+\partial_{n} v_{3}\right)\right] d s+\omega_{34} \\
& =\left[\sin \gamma \partial_{n} h+h \cos \gamma\left(h+\left(k_{n}-2 h\right)\right)\right] d s+\omega_{34} \\
& =\left[\sin \gamma \partial_{n} h-h \cos \gamma\left(h-k_{n}\right)\right] d s+\omega_{34} .
\end{aligned}
$$

The result then follows by applying Lemma 6.2 on page 1593 .

Proof of 1.1. If the surface intersects the plane $\left\{x_{3}=0\right\}$ in a constant angle, then $\gamma \equiv$ const. and we easily obtain

$$
\int_{\partial \Sigma} \partial_{n} h d s=0
$$


and

$$
\int_{\partial \Sigma} h\left(h-k_{n}\right) d s=0
$$

from (3.7) and (3.8).

Using a classical theorem of Joachimsthal we have that since the angle between the surface and the plane is constant along the boundary, the boundary is a line of curvature. Letting "prime" denote differentiation with respect to arc length on $\partial D$ we have

$$
\text { const. }=\langle X, v\rangle=-\left\langle X^{\prime \prime}, v\right\rangle=\left\langle X^{\prime}, v^{\prime}\right\rangle=-k_{n}
$$

and so $k_{n}$ is constant on $\partial D$.

Let $\lambda_{1}:=k_{n}$ and $\lambda_{2}:=2 h-k_{n}$ denote the principal curvatures on $\partial D$. Then (3.6) and (3.12) yield

$$
\int_{\partial D} \lambda_{1}-\lambda_{2} d s=0
$$

and

$$
\int_{\partial D} \lambda_{1}^{2}-\lambda_{2}^{2} d s=0
$$

By Hölder's inequality, we have for $j=1,2$

$$
\left|\int_{\partial D} \lambda_{j} d s\right| \leq \int_{\partial D}\left|\lambda_{j}\right| d s \leq\left(\int_{\partial D} \lambda_{j}^{2} d s\right)^{1 / 2}(2 \pi)^{1 / 2}
$$

However, since $\lambda_{1}=$ const. we have equality in (3.15) for $j=1$ and hence also for $j=2$ by (3.13) and (3.14). We can then conclude from the necessary condition for equality in Hölder's inequality, that $\lambda_{2} \equiv$ const. also. In particular $h \equiv$ const. holds on the boundary and by (3.13), every boundary point is an umbilic.

Let $I I_{Y}$ denote the second fundamental form of the map $Y$. As shown by Bryant [3], the form

$$
Q:=I I_{Y}^{(4,0)}
$$

defines a holomorphic quartic differential on any Willmore surface. A calculation similar to that in [15] shows that $Q$ is given locally in terms of a complex coordinate on $D$ by $Q=: q d z^{4}$ where

$$
q= \begin{cases}\left(\varphi^{2} / 4\right)\left(h^{2}+\Delta \log \varphi\right), & \text { if } \varphi \neq 0 \\ -\varphi_{z} h_{z}, & \text { if } \varphi=0\end{cases}
$$


We will need only the second part of this formula and it will be shown in the appendix. Here $\varphi d z^{2}$ is the Hopf differential which is the $(2,0)$ part of the second fundamental form of $X$. We recall that the Codazzi equations on $D$ take the form

$$
\varphi_{\bar{z}}=e^{\mu} h_{z}
$$

where $e^{\mu}|d z|^{2}$ is the local expression of the metric. Since the zeros of $\varphi$ correspond to umbilics, we have $\varphi \equiv 0$ on $\partial D$. Letting $z=r e^{i 9}$ denote the usual coordinate on $D$, we have on $\partial D$

$$
0=\partial_{\vartheta} \varphi=i\left(z \varphi_{z}-\bar{z} \varphi_{\bar{z}}\right)
$$

and

$$
0=\partial_{9} h=i\left(z h_{z}-\bar{z} h_{\bar{z}}\right) .
$$

Therefore on $\partial D$ we have, using (3.17), (3.18), and (3.19)

$$
\begin{aligned}
-q=\varphi_{z} h_{z}=\bar{z} z \varphi_{z} h_{z}=(\bar{z})^{2} \varphi_{\bar{z}} h_{z}=(\bar{z})^{2} e^{\mu} h_{z} h_{z} \\
=(\bar{z})^{3} e^{\mu} z h_{z} h_{z}=(\bar{z})^{4} e^{\mu} h_{z} h_{\bar{z}} .
\end{aligned}
$$

It then follows that the function $z^{4} q$ is holomorphic on the disc and is real valued on the boundary. By the maximum principle it follows that $z^{4} q \equiv a$ for some real constant $a$. Since $q=a / z^{4}$ is holomorphic in $D$ it then follows that $a=0$ and so $q \equiv 0$ holds in $D$. It then follows from results of [3] that either $X(D)$ is, after a conformal transformation, a minimal immersion or $X(D)$ is part of a sphere. Since the set of umbilics is a conformal invariant, we would in the first case obtain a minimal surface in $\mathbb{E}^{3}$ having a boundary component made up entirely of umbilics. It follows then that the surface is a flat disc since its Hopf differential vanishes identically. The only remaining possibility is that the surface is a spherical cap.

3.1. Remark. We wish to point out that the example of Babich and Bobenko mentioned in the introduction has the property that it cuts a horizontal plane in a constant angle along a circle of umbilics of the surface.

One might attempt to prove Theorem (1.1) as follows. Glue a spherical cap $C$ to $\Sigma$ along $S^{1}=\partial \Sigma$ so that the resulting closed surface $M$ is $C^{1}$. If one could succeed in showing that $M$ is of class $C^{4}$, then it would follow from Theorem 6.6.1 of [11] that $M$ is real analytic and hence that $M$ is a sphere since it coincides with a sphere along $C$. However this approach cannot work. Applying this idea to the Babich-Bobenko example yields a surface which is not only $C^{1}$ but is in fact $C^{2}$ along the circle. The argument above would then yield a contradiction if it were possible to prove that it is $C^{4}$. 


\section{EXAMPLES WITH NONCONSTANT ANGLE}

For $\rho>1$ let $u_{\rho}(x, y):=\left(\rho^{2}-x^{2}-y^{2}\right)^{1 / 2}-\left(\rho^{2}-1\right)^{1 / 2}$ so that the graph of $u_{\rho}$ over $\bar{D}$ is a spherical cap $C_{\rho}$ of radius $\rho$ bounded by $S^{1}$. We will show that $C_{\rho}$ can be perturbed to produce infinitely many Willmore surfaces bounded by $S^{1}$ which meets the plane of the circle in a nonconstant angle.

The mean curvature $h$ of the graph of a sufficiently smooth function $u$ is given by

$$
2 h=\operatorname{Div}\left(\frac{D u}{\sqrt{1+|D u|^{2}}}\right)
$$

where the divergence and gradient are computed using the metric in the plane. For a graph, Equation (1.1) can be expressed

$$
\begin{aligned}
0=F[u]:= & \left(\partial_{x}\left(\frac{1+u_{y}^{2}}{\sqrt{1+|D u|^{2}}} h_{x}\right)-\partial_{x}\left(\frac{u_{x} u_{y}}{\sqrt{1+|D u|^{2}}} h_{y}\right)\right. \\
& -\partial_{y}\left(\frac{u_{x} u_{y}}{\sqrt{1+|D u|^{2}}} h_{x}\right)+\partial_{y}\left(\frac{1+u_{x}^{2}}{\sqrt{1+|D u|^{2}}} h_{y}\right)+\cdots
\end{aligned}
$$

where $h$ is given by (4.20) and ... denotes lower order terms in $u$. The linearization of $F$ is the operator

$$
D F_{u}[w]:=\partial_{\varepsilon}(F[u+\varepsilon w])_{\varepsilon=0} .
$$

Note that $D F_{u_{\rho}}[$. ] can be considered as the Hessian at $u$ of the Willmore functional restricted to graphs so it is self-adjoint on the Sobolev space $W_{0}^{2,2}(D)$ with respect to the Euclidean $L^{2}$ product. Acting on this space, $D F_{u}$ [.] has a discrete spectrum $\mu_{1} \leq \mu_{2} \leq \mu_{3} \leq \ldots$.

Lemma 4.1. The first eigenvalue $\mu_{1}$ of the problem

$$
D F_{u_{\rho}}[w]=\mu w \text { in } D ; \quad w=0=\partial_{n} w \text { on } \partial D
$$

is strictly positive.

Proof. The integrand for the Willmore functional is non-negative and vanishes identically for a spherical cap so it is clear that $C_{\rho}$ minimizes the Willmore functional among all graphs over $D$ having boundary $S^{1}$ and meeting the plane in the same constant angle as $C_{\rho}$. From the interpretation of $D F_{u_{\rho}}[$.$] given above,$ the first eigenvalue of the problem (4.21) must be non-negative.

Suppose $\mu_{1}=0$ holds. Let $S$ be a 2 -sphere of radius $\rho$ containing $C_{\rho}$. Then $S$ minimizes the Willmore functional and is hence globally stable. Let $J$ denote the Jacobi operator for $W$ on $S$. $J$ acts on smooth function on $S$ thought of as the 
normal components of variation fields for smooth deformations of $S$. If $\mu_{1}=0$ holds, then the corresponding eigenfunction $w_{1}$ satisfies

$$
J\left[w_{1} v_{3}\right]=0, \quad \text { in } C_{\rho} ; \quad w_{1} v_{3}=0=\partial_{n}\left(w_{1} v_{3}\right) \quad \text { on } \partial C_{\rho}
$$

since $\left\langle w_{1} E_{3}, v\right\rangle=w_{1} v_{3}$. However, the first eigenvalue of $J$ is monotonically decreasing with respect to the domain, [17], so if $U$ is a relatively compact subdomain of $S$ containing $C_{\rho}$, than the first eigenvalue of $J$ in $U$ is negative, contradicting the stability of $S$.

Theorem 4.2. Let $g: \partial D \rightarrow \mathrm{R}$ be a function of Hölder class $C^{3+\alpha}, \alpha>0$. Then if $g$ is sufficiently close to to the constant function $-\left(\rho^{2}-1\right)^{-1 / 2}$ in $C^{3+\alpha}$, there exists a $C^{4}$ Willmore surface of disc type with circular boundary which makes an angle $\arccos \left(1+g^{2}\right)^{-1 / 2}$ with the plane along $\partial D$.

Proof. Define the Banach spaces $B_{1}=C^{4+\alpha}(D), B_{2}=C^{\alpha}(D) \times C^{4+\alpha}(\partial D) \times$ $C^{3+\alpha}(\partial D)$ and define

$$
\mathcal{F}: B_{1} \rightarrow B_{2}, \quad u \rightarrow\left(F[u],\left.u\right|_{\partial D}, \partial_{n} u\right) .
$$

Then $\mathcal{F}\left[u_{\rho}\right]=\left(0,0,-\left(\rho^{2}-1\right)^{-1 / 2}\right)$ and the derivative of $\mathcal{F}$ is

$$
\left.D \mathcal{F}\right|_{u_{\rho}}[w]=\left(D F_{u_{\rho}}[w],\left.w\right|_{\partial D}, \partial_{n} w\right) .
$$

It follows from Lemma (4.1) and Theorem 2.40 of [6], that, since the homogeneous equation $\left.D \mathcal{F}\right|_{u_{\rho}}=0$ has only the trivial solution, the linear boundary value problem, $\left.D \mathcal{F}\right|_{u_{\rho}}[w]=\left(f, g_{1}, g_{2}\right)$ is uniquely solvable in $B_{1}$ for all $\left(f, g_{1}, g_{2}\right) \in$ $B_{2}$. It then follows from the inverse function theorem that $\mathcal{F}$ is a $C^{1}$ diffeomorphism of a neighborhood of $u_{\rho}$ in $B_{1}$ onto a neighborhood of $\left(0,0,-\left(\rho^{2}-1\right)^{-1 / 2}\right)$ in $B_{2}$. From this the result easily follows.

\section{NATURAL BOUNDARY CONDITIONS}

Proposition 5.1. Let $X: \Sigma \rightarrow \mathbb{E}^{3}$ be a $C^{4}(\bar{\Sigma})$ immersion of an orientable compact surface with boundary. Then the first variation of the Willmore functional $\delta_{\dot{X}} W(X)$ vanishes for all variations satisfying $\left.\dot{X}\right|_{\partial \Sigma} \equiv 0$ if and only if there holds,

$$
\begin{gathered}
\Delta h+2 h\left(h^{2}-K\right)=0, \quad \text { in } \Sigma, \\
h \equiv k_{n}, \quad \text { on } \partial \Sigma .
\end{gathered}
$$

Proof. We write an arbitrary smooth variation of $X$ as $\dot{X}=\xi+\psi v$. where $\xi$ is tangent to $\Sigma$. In Section 6 we will show that the first first variation is given by

$$
\begin{gathered}
(5.24) \delta_{\dot{X}} W[X]=\int_{\Sigma} \psi\left(\Delta h+2 h\left(h^{2}-K\right)\right) d A+\oint_{\partial \Sigma}\left(h^{2}-K\right)\langle\xi, n\rangle-\psi \partial_{n} h d s \\
-\oint_{\partial \Sigma}\langle(d v+h 1) \nabla \psi, n\rangle d s,
\end{gathered}
$$


from which the result easily follows.

Theorem 5.2. Let $X:(D, \partial D) \rightarrow\left(\mathbb{E}_{+}^{3}, S^{1}\right)$ be a $C^{4}(\bar{D})$ embedding of a compact surface of disc type into the upper half space, which is a solution of (5.22) and (5.23). Then $X(D)$ is a spherical cap or a flat disc.

Proof. By considering $D$ with the conformal structure induced by $X$, we may assume that $X$ is a conformal immersion. Let $(r, \vartheta)$ be polar coordinates in $D-$ $\{0\}$ so that $(L, 9)$ are isothermal coordinates on $D-\{0\}$, where $L=\log r$. Note that $w:=\log z=L+i \vartheta$. We express the induced metric and second fundamental form, respectively by

$d s^{2}=: e^{2 \omega}\left(d L^{2}+d \vartheta^{2}\right), \quad I I=e^{2 \omega}\left(\sigma_{11} d L^{2}+\sigma_{12}(d L d \vartheta+d \vartheta d L)+\sigma_{22} d \vartheta^{2}\right)$.

Because of (5.23), we have $\sigma_{11}=h=\sigma_{22}$ on $\partial D$. The Frenet equations are,

$$
\begin{aligned}
& X_{99}=\omega_{9} X_{9}-\omega_{L} X_{L}+\sigma_{22} e^{2 \omega} v \\
& X_{9 L}=\omega_{L} X_{9}+\omega_{9} X_{L}+\sigma_{12} e^{2 \omega} v \\
& X_{L L}=-\omega_{9} X_{9}+\omega_{L} X_{L}+\sigma_{11} e^{2 \omega} v
\end{aligned}
$$

The holomorphic quartic differential $Q$ introduced in the Section 3 is given locally by

$$
Q=q d z^{4}=\tilde{q} d w^{4}=Y_{w w} \cdot Y_{w w} d w^{4} .
$$

Therefore

$$
\mathfrak{I} \tilde{q}=\frac{-1}{2}\left(Y_{L L}-Y_{9 \vartheta}\right) \cdot Y_{L \vartheta}
$$

Recall that for a Willmore surface, $Y$ is a harmonic, conformal map, so that $Y_{L L}+$ $Y_{99}=0,(\bmod Y)$ holds. It follows that

$$
\mathfrak{I} \tilde{q}=Y_{9 \vartheta} \cdot Y_{L \vartheta},
$$

since $Y_{L 9} \cdot Y=-Y_{L} \cdot Y_{9}=0$. We will use (5.28) to show that $Q \equiv 0$ holds on $D$.

Using that the boundary of the surface is the unit circle together with the given boundary conditions, it is easily found that,

$$
\begin{aligned}
h=k_{n} & =\left\langle E_{3}, n\right\rangle=-\langle X, v\rangle, \\
k_{g} & =\left\langle E_{3}, v\right\rangle=\langle X, n\rangle,
\end{aligned}
$$

hold where $k_{g}$ denotes the geodesic curvature of the boundary. Again, since the boundary is the unit circle, we have

$$
k_{n}^{\prime}=\sigma_{12} k_{g}, \quad k_{g}^{\prime}=-\sigma_{12} k_{n},
$$


where "prime" denotes differentiation with respect to arc length. The local expression for the geodesic curvature is $k_{g}=\omega_{L} e^{-\omega}$, so that we obtain,

$$
h_{9}=\sigma_{12} \omega_{L}, \quad\left(\omega_{L} e^{-\omega}\right)_{\vartheta}=-\sigma_{12} h e^{\omega},
$$

Combining the last two equations yields,

$$
h_{9 \vartheta}-\partial_{9}\left(\sigma_{12}\right) \omega_{L}-\left(\sigma_{12}\right) \omega_{L} \omega_{\vartheta}+h\left(\sigma_{12}\right)^{2} e^{2 \omega}=0 .
$$

Because of the boundary values, we have from (2.4),

$$
\left.Y\right|_{\partial D}=h\left(x_{1}, x_{2}, 0,0,1\right)+\left(v_{1}, v_{2}, v_{3},-h,-h\right),
$$

using (5.29). In particular $\left\langle Y, e_{5}\right\rangle \equiv 0$ holds on the boundary, where $\left\{e_{j}\right\}_{j=1, \ldots, 5}$, denotes the standard basis for the five dimensional Minkowski space $\mathbb{E}_{1}^{5}$. Using that $h=k_{n}$ along the boundary we get that the derivatives of $Y$ are given by,

$$
Y_{L}=h_{L} \underline{X}-\sigma_{12} \underline{X}_{9}, \quad Y_{9}=h_{\vartheta} \underline{X}-\sigma_{12} \underline{X}_{L},
$$

on the boundary. From the first equation, we see that $\left\langle Y_{L}, e_{4}\right\rangle \equiv 0$ on $\partial D$. It therefore follows that if, $\hat{Y}:=\left(Y_{1}, Y_{2}, Y_{3}\right)$, then $Y_{99} \cdot Y_{L 9}=\left\langle\hat{Y}_{99}, \hat{Y}_{L \vartheta}\right\rangle$, along the boundary.

Using the Frenet equations, (5.34) and (5.32), we compute,

$$
\begin{aligned}
& \hat{Y}_{99}=h_{99} X+\left[-\partial_{9}\left(\sigma_{12}\right)-\sigma_{12} \omega_{9}\right] X_{L}-\left(\sigma_{12}\right)^{2} e^{2 \omega} v . \\
& \hat{Y}_{L 9}=h_{L 9} X+\sigma_{12} \omega_{L} X_{L}-\sigma_{12} h e^{2 \omega} v, \quad\left(\bmod X_{9}\right) .
\end{aligned}
$$

Noting that along the boundary we have $\left\langle X_{L}, X\right\rangle=e^{\omega}\langle n, X\rangle=k_{g} e^{\omega}=\omega_{L}$, after a lengthy calculation using (5.29) we get

$$
\begin{aligned}
& \left\langle\hat{Y}_{9 \vartheta}, \hat{Y}_{L \vartheta}\right\rangle=h_{L \vartheta}\left[h_{9 \vartheta}-\omega_{L} \omega_{\vartheta} \sigma_{12}+h \sigma_{12}^{2} e^{2 \omega}\right] \\
& +\sigma_{12} \omega_{L} e^{2 \omega}\left[-\partial_{9}\left(\sigma_{12}-\sigma_{12} \omega_{\vartheta}\right]+\sigma_{12}^{3} h e^{4 \omega}+h_{9 \vartheta} \sigma_{12} \omega_{L}^{2}+h_{\vartheta \vartheta} \sigma_{12} e^{2 \omega} h^{2} .\right.
\end{aligned}
$$

We rewrite the last term, using that

$$
1=k_{n}^{2}+k_{g}^{2}=h^{2}+\left(\omega_{L} e^{-\omega}\right)^{2},
$$

on the boundary to obtain,

$$
\left\langle\hat{Y}_{9 \vartheta}, \hat{Y}_{L \vartheta}\right\rangle=\left(h_{L \vartheta}+\sigma_{12} e^{2 \omega}\right)\left[h_{9 \vartheta}-\omega_{L}\left(\sigma_{12}\right)_{9}-\omega_{L} \omega_{\vartheta} \sigma_{12}+h \sigma_{12}^{2} e^{2 \omega}\right]=0,
$$

by (5.33). 
We thus have by (5.26), that $\mathfrak{I}\left(z^{4} q\right) \equiv 0$ on $\partial D$, and so $z^{4} q \equiv r$, in $D$ for some real constant $r$. By the holomorphicity of $q$, this can only happen if $r=0$ and hence $q \equiv 0$ in $D$. By a theorem of Bryant, this implies that either the surface is already contained in a 2-sphere, or after a conformal transformation $F$, the surface is minimal. We need only consider the second possibility.

Let $\infty$ denote the point at infinity in $\mathbb{E}^{3}$. If $F^{-1}(\infty)$ does not lie on $X(\bar{D})$, then the surface is transformed into a compact minimal surface with circular, and hence planar boundary. It then follows from the maximum principle that the transformed surface is a flat disc and so the original surface was a disc or part of a sphere. If $F^{-1}(\infty)=X(p)$, with $p$ in the interior, then the boundary of $F \circ X$ is still a circle which we may assume, by rescaling, is the unit circle. Note that Equation (5.23) is conformally invariant since it is the boundary condition for a conformally invariant variational problem. Therefore, we have a minimal surface which has a circular boundary component along which $k_{n} \equiv 0$ holds. Then by (5.27), we can see that the angle between the surface and the plane of the circle is constant and so the circle is a circle of umbilic points of the surface. Since the umbilics on a nonflat minimal surface are isolated, the surface must be flat. The only remaining possibility is that $F^{-1}(\infty)$ lies on $X(\partial D)$. In this case $F \circ X(\partial D)$ is a line. A well known reflection principle for minimal surfaces can be applied to obtain a genus zero minimal surface with exactly one end. Since $F \circ X$ was assumed to be embedded in a half space, this genus zero minimal surface is embedded and has finite total curvature. By applying a theorem of Lopez and Ros, [9] one sees that the image of $F \circ X$ is a flat half plane. We may therefore conclude that the original surface was a spherical cap or a flat disc.

5.1. Remark. We have used the hypothesis that the surface is embedded in a half space in a very weak way and we believe that it can be removed.

\section{FIRST VARIATION OF THE FREE BOUNDARY PROBLEM}

Let $\Omega$ be a domain in $\mathbb{E}^{3}$ with smooth nonempty boundary. We let $\Sigma$ be a compact orientable surface with nonempty boundary and denote by $\mathcal{I}$ the space of all smooth $\left(C^{\infty}\right)$ immersions $(\Sigma, \partial \Sigma) \rightarrow(\Omega, \partial \Omega)$. Let $X \in \mathcal{I}$ and let $\varepsilon \rightarrow X_{\varepsilon}$ be a smooth curve in $\mathcal{I}$ defined on an interval containing 0 with $X_{0}=X$. If $\dot{X}:=\partial_{\varepsilon}\left(X_{\varepsilon}\right)_{\varepsilon=0}$ denotes the variation field, then we have $\langle\dot{X}, N\rangle \equiv 0$ on $\partial \Sigma$, where $N$ denotes the unit outward pointing normal to $\partial \Omega$. We will write $\dot{X}=\xi+\psi \nu$ where $\xi$ is a smooth vector field on $\Sigma, \psi$ is a smooth function and $v$ denotes is a unit normal to $\Sigma$. Let $t$ and $n$ denote the unit tangent and the unit outward normal to $\partial \Sigma$ so that along the boundary $e_{1}=n, e_{2}=t, e_{3}=v$ defines a positively oriented frame. Let $\sigma_{i j}:=-\left\langle d v\left(e_{i}\right), e_{j}\right\rangle$.

Proposition 6.1. $\delta_{\dot{X}} W[X] \equiv 0$ for every variation of $X$ in $\mathcal{I}$ if and only if

$$
\Delta h+2 h\left(h^{2}-K\right)=0, \text { on } \Sigma
$$




$$
\begin{gathered}
\sigma_{11} \equiv h \equiv \sigma_{22} \text {, on } \partial \Sigma \\
\left(h^{2}-K\right)\langle v, N\rangle+\left(\partial_{n} h+\partial_{t} \sigma_{12}\right)\langle n, N\rangle \equiv 0 \text {, on } \partial \Sigma .
\end{gathered}
$$

Two obvious examples are:

(i) $\partial \Omega$ is a plane and $\Sigma$ is a hemisphere meeting the plane in a right angle,

(ii) $\partial \Omega$ is a plane or sphere and $\Sigma$ is a spherical cap or disc meeting $\partial \Omega$ in a right angle.

\section{Lemma 6.2.}

$$
\delta_{\psi v} \int_{\Sigma} K d A=\oint_{\partial \Sigma}\langle(d v+2 h 1) \nabla \psi, n\rangle d s .
$$

Proof. We will show that the pointwise variation of $K$ is given by

$$
\delta_{\psi v} K=\left\langle d v+2 h 1, \nabla^{2} \psi\right\rangle+2 h K \psi .
$$

We assume this for the moment. Then

$$
\delta_{\psi v} \int_{\Sigma} K d A=\int_{\Sigma}\left(\delta_{\psi v} K\right)-2 h \psi K d A=\int_{\Sigma}\left\langle d v+2 h 1, \nabla^{2} \psi\right\rangle d A .
$$

However using the Codazzi equations, one can show that

$$
\operatorname{div}((d v+2 h 1) \nabla \psi)=\sum_{i=1,2} e_{i}\left\langle(d v+2 h 1) \nabla \psi, e_{i}\right\rangle=\left\langle d v+2 h 1, \nabla^{2} \psi\right\rangle
$$

holds and so (6.40) follows by Stoke's theorem.

We now prove (6.41). Fix $p \in \Sigma$ and let $e_{i}, i=1,2$ be an orthonormal frame near $p$ (with respect to the metric induced by $X$ ) which diagonalizes the second fundamental form at $p$, i.e. $d v=-k_{i} e_{i}, i=1,2$, where $k_{i}$ are the principle curvatures. For the variation $X_{\varepsilon}=X+\varepsilon \psi v+O\left(\varepsilon^{2}\right)$ let $g_{i j}=g_{i j}(\varepsilon), L_{i j}=L_{i j}(\varepsilon)$ be the components of the first and second fundamental forms of $X_{\varepsilon}$ with respect to this frame. Let $g=\operatorname{det}\left(g_{i j}\right), L=\operatorname{det}\left(L_{i j}\right)$ so that $K=K(\varepsilon)=L / g$ holds. Since $g(0)=1$ and $\dot{g}(0)=-4 h \psi$, we obtain

$$
\delta_{\psi v} K=\dot{L}+4 h K \psi
$$

Using that the corresponding variation of the normal is given by $\dot{v}=-\nabla \psi$ one can easily obtain that

$$
\dot{L}_{i j}=\left\langle\nabla_{e_{i}} \nabla \psi, e_{j}\right\rangle-\psi\left\langle d v\left(e_{i}\right), d v\left(e_{j}\right)\right\rangle
$$

from which one obtains $\dot{L}=\left\langle d v+2 h 1, \nabla^{2} \psi\right\rangle-2 h K \psi$. Finally, (6.41) follows from (6.42). 
Proof of Proposition 6.1. We begin by writing $\dot{X}=\xi+\psi v$ with $\xi$ tangent to $\Sigma$. We have

$$
\left.\delta_{\xi} W[X]=\int_{\Sigma} \mathcal{L}_{\xi}\left[\left(h^{2}-K\right) d A\right]=\int_{\Sigma} d(\xi]\left[\left(h^{2}-K\right) d A\right]=\oint_{\partial \Sigma}\left(h^{2}-K\right) \xi\right\rfloor d A .
$$

(Here $\mathcal{L}$ denotes the Lie derivative and $\rfloor$ denotes the interior product of a vector field with a differential form). We obtain,

$$
\delta_{\xi} W[X]=\oint_{\partial \Sigma}\left(h^{2}-K\right)\langle\xi, n\rangle d s .
$$

We will need the well known formulas,

$$
\delta_{\psi v} d A=-2 h \psi d A, \quad \delta_{\psi v} h=1 / 2\left(\Delta \psi+\left(4 h^{2}-2 K\right) \psi\right) .
$$

We compute

$$
\begin{aligned}
\delta_{\psi v} \int_{\Sigma}\left(h^{2}-K\right) d A \\
=\int_{\Sigma}\left(2 h \delta_{\psi v} h-2 h^{2} \psi\right) d A-\oint_{\partial \Sigma}\langle(d v+2 h 1) \nabla \psi, n\rangle d s \\
=\int_{\Sigma} h\left(\Delta \psi+2 \psi\left(h^{2}-K\right)\right) d A-\oint_{\partial \Sigma}\langle(d v+2 h 1) \nabla \psi, n\rangle d s \\
=\int_{\Sigma} \psi\left(\Delta h+2 h\left(h^{2}-K\right)\right) d A+\oint_{\partial \Sigma}\left(h \partial_{n} \psi-\psi \partial_{n} h\right) d s \\
\quad-\oint_{\partial \Sigma}\langle(d v+2 h 1) \nabla \psi, n\rangle d s \\
=\int_{\Sigma} \psi\left(\Delta h+2 h\left(h^{2}-K\right)\right) d A-\oint_{\partial \Sigma} \psi \partial_{n} h d s-\oint_{\partial \Sigma}\langle(d v+h 1) \nabla \psi, n\rangle d s .
\end{aligned}
$$

Combining the last line with (6.43) and simplifying gives,

$$
\begin{aligned}
\delta_{\dot{X}} W[X]=\int_{\Sigma} \psi\left(\Delta h+2 h\left(h^{2}-K\right)\right) d A+\oint_{\partial \Sigma} & \left(h^{2}-K\right)\langle\xi, n\rangle-\psi \partial_{n} h d s \\
& -\oint_{\partial \Sigma}\langle(d v+h 1) \nabla \psi, n\rangle d s .
\end{aligned}
$$

First, by considering an arbitrary variation with $\psi \in C_{c}^{\infty}(\Sigma)$ and $\xi \equiv 0$, we obtain (6.37). To obtain (6.38), we solve the biharmonic Dirichlet problem

$$
\begin{gathered}
\Delta^{2} \psi=0, \text { in } \Sigma \\
\psi=0, \partial_{n} \psi=h-\sigma_{11}, \text { on } \partial \Sigma .
\end{gathered}
$$


Let $\dot{X}=\psi v$ and note that $\langle\dot{X}, N\rangle \equiv 0$ holds on $\partial \Sigma$. Since $\nabla \psi=\left(h-\sigma_{11}\right) n$ on $\partial \Sigma$, we obtain

$$
\delta_{\dot{X}} W[X]=-\oint_{\partial \Sigma}\left(h-\sigma_{11}\right)\langle(d v+h 1) n, n\rangle d s=-\oint_{\partial \Sigma}\left(h-\sigma_{11}\right)^{2} d s .
$$

so that $\sigma_{11} \equiv h$ holds on $\partial \Sigma$. The other equality in (6.38) follows from $h:=$ $(1 / 2)\left(\sigma_{11}+\sigma_{22}\right)$.

Assuming that (6.37) and (6.38) hold, we write $\left.(\nabla \psi)\right|_{\partial \Sigma}=\psi_{t} t+\psi_{n} n$ and so

$$
\begin{aligned}
\delta_{\dot{X}} W[X] & =\oint_{\partial \Sigma}\left(h^{2}-K\right)\langle\xi, n\rangle-\psi \partial_{n} h d s-\oint_{\partial \Sigma}\left\langle(d v+h 1) \partial_{t}(\psi) t, n\right\rangle d s \\
& =\oint_{\partial \Sigma}\left(h^{2}-K\right)\langle\xi, n\rangle-\psi \partial_{n} h d s+\oint_{\partial \Sigma} \sigma_{12} \partial_{t}(\psi) d s .
\end{aligned}
$$

Integrating by parts then gives

$$
\delta_{\dot{X}} W[X]=\oint_{\partial \Sigma}\left(h^{2}-K\right)\langle\xi, n\rangle-\psi\left(\partial_{n} h+\partial_{t} \sigma_{12}\right) d s .
$$

We claim that $\delta_{\dot{X}} W[X]=0$ holds for all variations satisfying

$$
0 \equiv\langle\dot{X}, N\rangle=\langle\xi, n\rangle+\psi\langle v, N\rangle
$$

if and only if (6.39) holds.

Let $j$ denote $90^{\circ}$ rotation in the plane $\operatorname{span}\{n, v\}$, i.e. $j n=v, j v=-n$. Let $V:=\left(\partial_{n} h+\partial_{t} \sigma_{12}\right) n+\left(h^{2}-K\right) v$. Then (6.39) can be written as $\langle V, N\rangle \equiv 0$. Note that the integrand appearing in (6.44) is $-\langle\dot{X}, j V\rangle$. If $\langle\dot{X}, N\rangle=0$ holds at each boundary point then the projection of $\dot{X}$ to $\operatorname{span}\{n, v\}$ is proportional to $j N$ and hence $-\langle\dot{X}, j V\rangle \sim\langle j N, j V\rangle \equiv 0$ and so the integral in (6.44) vanishes.

Conversely, suppose that (6.39) does not hold. We may assume then that $\langle V, N\rangle>0$ holds on some arc $\alpha \subset \partial \Sigma$. Let $\zeta$ be a smooth, nonnegative function on $\partial \Sigma$ with support in $\alpha$. Let $\dot{X}$ be a smooth extension of $\zeta j N$ to $\Sigma$. Then $\langle\dot{X}, N\rangle \equiv 0$ holds and $\delta_{\dot{X}} W \neq 0$ by (6.44).

It is also reasonable to consider a type of partitioning problem for Willmore surfaces in the spirit of [12]. We let $\Omega$ be a compact domain in $\mathbb{E}^{3}$ with smooth boundary $\partial \Omega$ which is almost everywhere immersed. We consider critical points of the Willmore functional $W$ among all sufficiently smooth surfaces $\Sigma \subset \Omega$ with $\partial \Sigma \subset \partial \Omega$ and which divide $\Omega$ into two domains of fixed volumes $V_{1}$ and $V_{2}$. Using Lagrange multipliers, one sees that we are seeking critical points of the functional

$$
W_{\lambda}=W-\lambda V
$$


where $V$ denotes the algebraic volume between one side of $\Sigma$ and $\partial \Omega$ and $\lambda$ is a Lagrange multiplier.

The set of allowable variations will then be given by those $\dot{X}=\xi+\psi v$ with $\langle\dot{X}, N\rangle \equiv 0$ on $\partial \Sigma$ and satisfying

$$
\int_{\Sigma} \psi d A=0
$$

The Euler-Lagrange equations for this problem are given by

$$
\begin{gathered}
\Delta h+2 h\left(h^{2}-K\right)=\lambda \text { on } \Sigma \\
\sigma_{11} \equiv h \equiv \sigma_{22} \text { on } \partial \Sigma \\
\left(h^{2}-K\right)\langle\nu, N\rangle+\left(\partial_{n} h+\partial_{t} \sigma_{12}\right)\langle n, N\rangle \equiv 0 \text { on } \partial \Sigma .
\end{gathered}
$$

\section{A UNIQUENESS THEOREM FOR THE FREE BOUNDARY PROBLEM}

In this section we will prove a uniqueness theorem for disc type Willmore surfaces in a half space which are critical for the problem posed in the previous section. The analogous result for the area functional was obtained by Nitsche in [13]. $D$ will denote the unit disc. $\mathbb{E}^{2} \subset \mathbb{E}^{3}$ will denote the plane given by $x_{3}=0$. We begin with a result about the more general system (6.45)-(6.47).

Proposition 7.1. Let $X:(\Sigma, \partial \Sigma) \rightarrow\left(\mathbb{E}_{+}^{3}, \mathbb{E}^{2}\right)$ be a $C^{4}(\bar{\Sigma})$ embedding of a compact surface which is a solution of (6.45)-(6.47). Then $\lambda \leq 0$ holds.

7.1. Remark. We wish to emphasize that in this result we are assuming that the surface is embedded up to the boundary, while in the following result we are assuming that the surface is immersed up to the boundary. It would be interesting to know if this amount of regularity could be shown to hold a priori.

Proof. In this case the condition (6.47) becomes

$$
\left(h^{2}-K\right)\left\langle v, E_{3}\right\rangle+\left(\partial_{n} h+\partial_{t} \sigma_{12}\right)\left\langle n, E_{3}\right\rangle \equiv 0 \text {, on } \partial \Sigma .
$$

Since the coordinate function $x_{3}$ on $\Sigma$ assumes its minimum $x_{3}=0$ on $\partial \Sigma$, we have $0 \geq \partial_{n} x_{3}=\left\langle n, E_{3}\right\rangle$ on $\partial \Sigma$. Since $h^{2}-K \geq 0$ holds, it follows that $\left\langle v, E_{3}\right\rangle$ and $\left(\partial_{h} h+\partial_{t} \sigma_{12}\right)$ must have the same sign, i.e. that

$$
\left(\partial_{n} h+\partial_{t} \sigma_{12}\right)\left\langle v, E_{3}\right\rangle \geq 0
$$

holds on $\partial \Sigma$. Partial integration then gives

$$
\begin{aligned}
0 & \leq \oint_{\partial \Sigma}\left(\partial_{n} h+\partial_{t} \sigma_{12}\right)\left\langle v, E_{3}\right\rangle d s=\oint_{\partial D} \partial_{n} h\left\langle v, E_{3}\right\rangle-\sigma_{12} \partial_{t}\left\langle v, E_{3}\right\rangle d s \\
& =\oint_{\partial D}\left(\partial_{n} h\right)\left\langle v, E_{3}\right\rangle+\sigma_{12}^{2}\left\langle n, E_{3}\right\rangle d s .
\end{aligned}
$$


We next compute the first variation of the Willmore functional for $\dot{X}=E_{3}$. Although this variation does not satisfy the boundary or volume constraints, the first variation must vanish since the variation is one through isometries of $\mathbb{E}^{3}$. We write $E_{3}=:\left\langle v, E_{3}\right\rangle v+E_{3}^{T}$ where the second term is the tangential component of $E_{3}$. Then using the results of the previous section, we obtain

$$
\begin{aligned}
0=\delta_{E_{3}} W= & -\lambda \int_{\Sigma} v_{3} d A+\oint_{\partial \Sigma}\left(h^{2}-K\right)\left\langle E_{3}^{T}, n\right\rangle-\left\langle v, E_{3}\right\rangle \partial_{n} h d s \\
& -\oint_{\partial \Sigma}\left\langle(d v+h 1) \nabla\left\langle v, E_{3}\right\rangle, n\right\rangle d s \\
= & -\lambda \int_{\Sigma} v_{3} d A+\oint_{\partial \Sigma}\left(h^{2}-K\right)\left\langle E_{3}^{T}, n\right\rangle-\left\langle v, E_{3}\right\rangle \partial_{n} h d s \\
-\oint_{\partial \Sigma}\left\langle\nabla\left\langle v, E_{3}\right\rangle,(d v+h 1) n\right\rangle d s & \\
=-\lambda \int_{\Sigma} v_{3} d A+\oint_{\partial \Sigma}\left(h^{2}-K\right)\left\langle E_{3}^{T}, n\right\rangle-\left\langle v, E_{3}\right\rangle \partial_{n} h d s & +\oint_{\partial \Sigma} \sigma_{12} \partial_{t}\left\langle v, E_{3}\right\rangle d s \\
= & -\lambda \int_{\Sigma} v_{3} d A+\oint_{\partial \Sigma}\left(h^{2}-K\right)\left\langle E_{3}^{T}, n\right\rangle-\left\langle v, E_{3}\right\rangle \partial_{n} h d s \\
& -\oint_{\partial \Sigma} \sigma_{12}^{2}\left\langle n, E_{3}\right\rangle d s \\
= & -\lambda \int_{\Sigma} v_{3} d A-\oint_{\partial \Sigma}\left\langle v, E_{3}\right\rangle \partial_{n} h d s,
\end{aligned}
$$

using the identity $h^{2}-K=h^{2}-\left(\sigma_{11} \sigma_{22}-\sigma_{12}^{2}\right)=\sigma_{12}^{2}$ on $\partial \Sigma$ by (6.38). Let $G$ denote the area in the plane $x_{3}=0$ enclosed by $\partial \Sigma$. Applying the divergence theorem to the constant vector $E_{3}$ gives

$$
\int_{\Sigma} v_{3} d A=G
$$

We therefore obtain from (7.48), (7.49) and (7.50)

$$
-\lambda G+\oint_{\partial D} \sigma_{12}^{2}\left\langle n, E_{3}\right\rangle d s=0
$$

Since $\partial_{n} x_{3}=\left\langle n, E_{3}\right\rangle \leq 0$ holds on $\partial D$, this implies that $\lambda \leq 0$ holds.

Theorem 7.2. Let $X:(D, \partial D) \rightarrow\left(\mathbb{E}_{+}^{3}, \mathbb{E}^{2}\right)$ be a $C^{4}(\bar{D})$ immersion which is a solution of (6.37)-(6.39). Then $X(D)$ is either a spherical cap or flat disc meeting the plane in a constant angle. 
Proof. Since $\lambda=0$ for a Willmore surface, we obtain from (7.51), that $\sigma_{12}^{2}\left\langle n, E_{3}\right\rangle \equiv 0$ holds on $\partial D$. If $\left\langle E_{3}, n\right\rangle \equiv 0$ holds on any open arc of the boundary, then $\sigma_{12}$ also vanishes on the arc by Joachimsthal's theorem and so by continuity $\sigma_{12} \equiv 0$ holds on $\partial D$. By (6.38) this means that the boundary is composed entirely of umbilic points. Note that up to now we have not used the fact that the surface is a topological disc. We are now in a situation which is very close to that in Theorem (1.1) except that we do not have the assumption that the boundary is circular. We will continue by using the coordinates and notation introduced in Section 5. Again we will use (5.28) to show that the quartic differential $Q$ vanishes identically.

Since $0 \equiv h^{2}-K=\sigma_{12}^{2}$ on $\partial D$, (6.39) gives $h_{n}\left\langle E_{3}, n\right\rangle \equiv 0$ on $\partial D$. On any open arc in $\partial D$ where $\left\langle E_{3}, n\right\rangle \equiv 0$ holds, we have that $v \equiv E_{3}$ on that arc and so $0 \equiv h=\sigma_{22}=-\left\langle\partial_{t} v, t\right\rangle=-\left\langle\partial_{t} E_{3}, t\right\rangle$ on the arc, using (6.38). It then easily follows that $h_{t} h_{n} \equiv 0$ on $\partial D$. We again appeal to the appendix where it is shown that $h_{t} h_{n}$ vanishes if and only if $\mathfrak{I} \tilde{q}$ vanishes. However by the transformation rule for a holomorphic quartic differential, $\tilde{q}=z^{4} q$ so we obtain that $\mathfrak{J}\left(z^{4} q\right) \equiv 0$ on $\partial D$ and hence by the maximum principle $z^{4} q \equiv a$ on $D$ for some real constant a. Since $q$ is holomorphic on all of $D$ it then follows that $q \equiv 0$ must hold on $D$. We can then finish the proof in the same way as in Theorem (1.1) by using Bryant's theorem to conclude that the immersion must be either totally umbilic or minimal. The non-flat minimal case is excluded since the boundary contains an arc of umbilics.

7.2. Remark. If no restriction on the genus of the surface is made then it is clear from the proof that one can still obtain that the boundary consists entirely of umbilics. Since the geodesic torsion along a curve of umbilics vanishes identically, it then follows that the surface must cut the plane in a constant angle. The reference [2] produces examples of compact Willmore surfaces in a half space which are not totally umbilic and which meet the plane in an angle of $90^{\circ}$. It is easy to see using Joachimsthal's theorem that these surfaces solve (6.37)-(6.39).

7.3. Remark. Using K.Brakke's Surface Evolver program, we have generated a picture (Figure 1) of a possible genus one solution of the free boundary problem(6.37)-(6.39).

Corollary 7.3. Let $B$ denote the unit ball in $\mathbb{E}^{3}$ and let Let $X:(D, \partial D) \rightarrow$ $\left(B, S^{2}\right)$ be a $C^{4}(\bar{D})$ immersion which is a solution of (6.37)-(6.39) with $N=X$. Then $X(D)$ is either a spherical cap or flat disc meeting $S^{2}$ in a constant angle.

Proof. The result follows from the theorem by using a suitable conformal map of $B$ into the half space $\mathbb{E}_{+}^{3}$. 


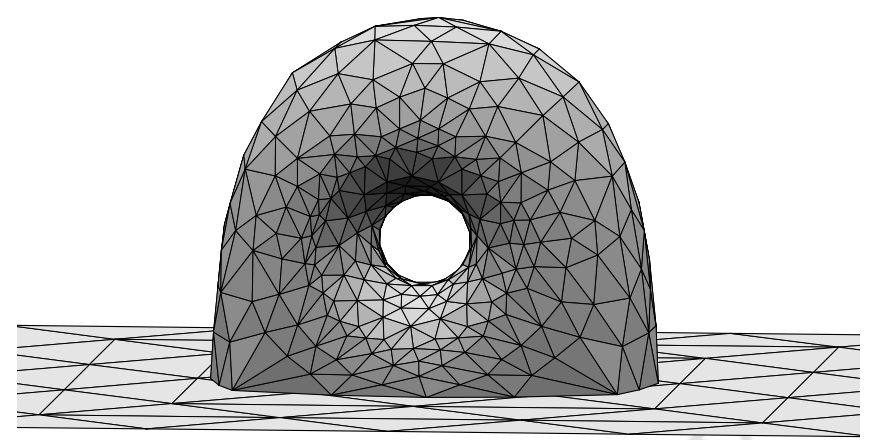

FIGURE 1

8. APPENDIX

We prove the local expression for $q$ used above. We have

$$
\begin{aligned}
Y & =h\left(X, \frac{X^{2}-1}{2}, \frac{X^{2}+1}{2}\right)+(\nu,\langle X, v\rangle,\langle X, v\rangle) \\
& =: h \underline{X}+(v, s, s) .
\end{aligned}
$$

Recall that $\cdot$ denotes the metric in $\mathbb{E}_{1}^{5}$ and note that $\underline{X} \cdot \underline{X} \equiv 0$ holds. We compute

$$
\begin{aligned}
Y_{z} & =h_{z} \underline{X}+h \underline{X}_{z}+\left(v_{z}, s_{z}, s_{z}\right) \\
& =h_{z} \underline{X}+h\left(X_{z},\left\langle X_{z}, X\right\rangle,\left\langle X_{z}, X\right\rangle\right)+\left(v_{z},\left\langle X, v_{z}\right\rangle,\left\langle X, v_{z}\right\rangle\right) .
\end{aligned}
$$

Using the Weingarten equation

$$
v_{z}=-h X_{z}-\varphi e^{-\mu} X_{\bar{z}}
$$

we obtain

$$
Y_{z}=h_{z} \underline{X}-\varphi e^{-\mu}\left(X_{\bar{z}},\left\langle X_{\bar{z}}, X\right\rangle,\left\langle X_{\bar{z}}, X\right\rangle\right)
$$

At an umbilic we have $\varphi=0$ and so

$$
Y_{z z}=h_{z z} \underline{X}+h_{z}\left(X_{z},\left\langle X_{z}, X\right\rangle,\left\langle X_{z}, X\right\rangle\right)-\varphi_{z} e^{-\mu}\left(X_{\bar{z}},\left\langle X_{\bar{z}}, X\right\rangle,\left\langle X_{\bar{z}}, X\right\rangle\right)
$$

and hence at an umbilic we have

$$
Y_{z z} \cdot Y_{z z}=-h_{z} \varphi_{z}
$$


In terms of the coordinate $w=\log z$, we express the induced metric $d s^{2}=$ : $e^{2 \omega}|d w|^{2}$ and the second fundamental form as $I I=\mathfrak{R}\left\{\tilde{\varphi} d w^{2}+h e^{2 \omega} d w d \bar{w}\right\}$. By the calculation above,

$$
\tilde{q}=-\tilde{\varphi}_{w} h_{w} .
$$

We can compute the first and second derivatives of $Y$ along the boundary using 6.38 on page 1593 and the fact that $\tilde{\varphi} \equiv 0$ on the boundary to obtain,

$$
\begin{array}{cl}
Y_{L}=h_{L} \underline{X}, & Y_{L \vartheta}=h_{L \vartheta} \underline{X}+h_{L} \underline{X}_{\vartheta} \\
Y_{\vartheta}=h_{\vartheta} \underline{X}, & Y_{\vartheta \vartheta}=h_{\vartheta \vartheta} \underline{X}+h_{\vartheta} \underline{X}_{9} .
\end{array}
$$

Therefore

$$
Y_{L \vartheta} \cdot Y_{9 \vartheta}=h_{L} h_{\vartheta} e^{2 \omega}=h_{n} h_{t} e^{4 \omega} .
$$

\section{REFERENCES}

[1] L. Alías, R. López \& B. Palmer, Stable constant mean curvature surfaces with circular boundaries, Amer. Math. Soc. Proc. 127 (1999), 1195-2000.

[2] M. BABICH \& A. BOBENKO, Willmore tori with umbilic lines and minimal surfaces in hyperbolic space, Duke Math. J. 72 (1993), 141-185.

[3] R. BRyant, A duality theorem for Willmore surfaces, J. Differential Geom. 20 (1984), 23-53.

[4] R. Courant \& D. HilberT, Methods of Mathematical Physics, Volume I, John Wiley, New York, 1989.

[5] R. EARP, B. BRito, W.H. MeEks, H. RosenberG, Structure theorems for constant mean curvature surfaces bounded by a planar curve, Indiana Univ. Math. J. 40 (1991), 333-343.

[6] Y. V. Egorov \& M. A. Shubin (Editors), Partial Differential Equations, in the series Encyclopedia of Mathematical Sciences, Vol. 30, Springer Verlag 1992, Berlin.

[7] N. KAPOUlEAS, Compact constant mean curvature surfaces in Euclidean 3-space, J. Diff. Geom. 33 (1991), 683-715.

[8] N. KorevaAR, R. Kusner, \& B. SOlOMOn, The structure of complete embedded surfaces with constant mean curvature, J. Diff. Geom. 30 (1989), 465-503.

[9] F. LOPEZ \& A. Ros, Embedded complete minimal surfaces of genus zero, J. Diff. Geom. 33 (1991), 293-300.

[10] R. LÓPEZ \& S. Montiel, Constant mean curvature surfaces with planar boundary, Duke Math. J. 85 (1996), 583-604.

[11] C. MOrReY, Multiple Integrals in the Calculus of Variations, Grundlehren Series Volume 130, Springer-Verlag, Berlin, (1966).

[12] JOHAnnes C.C. NitsCHE, Stationary partitioning of convex bodies, Arch. Rational Mech. and Anal. 89 (1985), 1-19.

[13] JOHANNES C.C. NiTSCHE, Boundary value problems for variational integrals involving surface curvatures, Quaterly of Applied Mathematics, Vol. LI, No. 2 (1993), 363-387.

[14] JOHANNES C.C. NitSCHE, Periodic surfaces that are extremal for energy functionals containing curvature functions. In: Statistical theromodynamics and differential geometry of microstructured materials (Minneapolis, MN, 1991) 69-98, IMA Vol. Math. Appl. 51 , Springer, New York, 1993.

[15] B. PALMER, The conformal Gauss map and the stability of Willmore surfaces, Ann. Global Analysis Geom. 9 (1991), 305-317.

[16] S. D. Poisson, Mémoire sur les surfaces élastiques, Cl. Sci. Mathém. Phys. Inst. de France (1833) 223-331.

[17] S. SMale, On the Morse Index Theorem, J. Math. Mech. 14 (1965), 1049-1055. 
[18] G. Thomsen, Über konforme Geometrie I: Grundlagen der konformen Flächentheorie. Abh. Math. Sem. Univ. Hamburg (1923), 31-56.

[19] H. Wente, The symmetry of sessile and pendent drops, Pac. J. Math. 88 (1980), 387-397

Department of Mathematical Sciences

University of Durham

Durham DH1 3LE, England

E-MAIL: bennett.palmer@durham.ac.uk

KEY WORDS AND PHRASES: Willmore surface, flux formula, free boundary. 1991 MATHEMATICS SUBJECT ClassifiCATION: 53A10.

Received: September 28th, 1999; revised: May 22nd, 2000. 